\title{
X-RAY AND NEUTRON SCATTERING STUDIES OF COMPLEX CONFINED FLUIDS
}

\author{
SUNIL K. SINHA \\ Advanced Photon Source \\ Argonne National Laboratory \\ Argonne, Illinois 60439
}

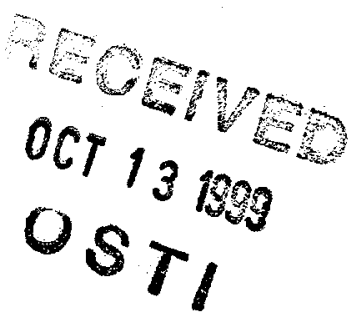

We review recent X-ray and neutron scattering studies of the structure and dynamics of confined complex fluids. This includes the study of polymer conformations and binary fluid phase transitions in porous media using Small Angle Neutron scattering, and the use of synchrotron radiation to study ordering and fluctuation phenomena at solid/liquid and liquid/air interfaces. Ordering of liquids near a solid surface or in confinement will be discussed, and the study, via specular and off-specular X-ray reflectivity, of capillary wave fluctuations on liquid polymer films. Finally, we shall discuss the use of highbrilliance beams from $X$-ray synchrotrons to study via photon correlation spectroscopy the slow dynamics of soft condensed matter systems.

\section{Introduction}

The behavior of fluids in confinement often differs from their bulk behavior in interesting ways. Thus fluids confined in a microporous medium for instance may have their phase behavior relative to melting or freezing modified. In other cases, the way in which a binary fluid system mixes or demixes may be modified. To some extent, a rather simple (and consequently less interesting) effect of confinement is simply a shift of various regions of the phase diagram with regard to temperature or concentration. However, more interestingly, in other cases the behavior itself may be modified. In particular, one may ask the question in the case of a second-order (continuous) phase transition, such as at a consolute point for a binary liquid mixture or a liquid/gas critical point, how is the critical behavior altered when the fluid is confined in micropores?

One may also ask whether the behavior is affected by the confinement or by the randomness of the pore structure. In both cases the effect is due to interaction with the pore walls, but considerations of randomness led a number of people a few years ago to propose the exciting idea that, for instance, a binary fluid demixing transition (which has a 3D-Ising-like critical behavior in bulk) maps on to the Random Field Ising Model (RFIM). The conformation of polymers confined in porous media also differs from that 


\section{DISCLAIMER}

This report was prepared as an account of work sponsored by an agency of the United States Government. Neither the United States Government nor any agency thereof, nor any of their employees, make any warranty, express or implied, or assumes any legal liability or responsibility for the accuracy, completeness, or usefulness of any information, apparatus, product, or process disclosed, or represents that its use would not infringe privately owned rights. Reference herein to any specific commercial product, process, or service by trade name, trademark, manufacturer, or otherwise does not necessarily constitute or imply its endorsement, recommendation, or favoring by the United States Government or any agency thereof. The views and opinions of authors expressed herein do not necessarily state or reflect those of the United States Government or any agency thereof. 


\section{DISCLAIMER}

Portions of this document may be illegible in electronic image products. Images are produced from the best available original document. 
in free solution in ways which depend on both the concentration and the ratio of polymer size to pore size, as has been discussed theoretically by DeGennes, Brochard and others. Important structural aspects of all of the above phenomena can be studied by small angle neutron scattering. This technique has the advantage that it can study concentration or density fluctuations inside media opaque to light and at short length scales which can vary from a few nanometers to microns. In addition, the techniques of contrast matching into selective deuteration of the sample can simplify the interpretation of the results.

This is because it is possible to fill the pores with a fluid whose scattering density exactly matches that of the solid, thus removing the scattering from the pore structure itself, thereby reducing the 2-phase system to a 1-phase system. The only fluctuations which will then scatter radiation are deviations from the average density, which will be fluctuations in the fluid itself.

Besides being of basic scientific interest, the behavior of fluids or polymers in porous materials is of considerable importance for processes such as enhanced oil recovery, chemical processing, and chemical or biological separation or filtration processes involving porous membranes or gels. The transport of fluids and polymers inside porous materials is also of considerable interest, but can only be indirectly studied by neutron or X-ray scattering. However, recent application of coherent X-ray beams from the new synchrotron radiation sources can make it possible to study the dynamics of such processes by using photon intensity correlation spectroscopy, as will be discussed at the end of these lectures.

One may of course also consider fluids confined at a surface or an interface or in a thin film. Here again, there are interesting predictions and observations regarding differences from bulk behavior. Scattering experiments such as specular and off-specular reflection of neutrons and $\mathrm{X}$-rays (in particular the later, where one may now exploit the high brilliance of $\mathrm{X}$-ray beams from synchrotron radiation sources) can elucidate effects such as crystallization or melting phenomena at interfaces, fluctuations due to capillary waves, etc., and we shall discuss some of these experiments also in these lectures.

\section{Small Angle Scattering Studied of Fluids Confined in Porous Media}

The basic methodology of small-angle scattering has been discussed in a variety of excellent reviews [1-4]. Neglecting any inelasticity in the scattering, the number of particles per second scattered by a sample into a detector is given by

$$
\mathrm{I}=\mathrm{S}(\vec{q})\left(I_{o} / A\right)(\Delta \Omega)
$$

where $I_{0}$ is the number of particles per second in the incident beam of cross-sectional area $A,(\Delta \Omega)$ is the solid angle subtended at the sample by the detector, $S(\vec{q})$ is the scattering function characterizing the sample and $\vec{q}$ is the so-called wavevector transfer defined by 


$$
\vec{q}=\bar{k}_{1}-\bar{k}_{0}
$$

where $\vec{k}_{0}, \vec{k}_{1}$ are the wavevectors of the incident and scattered radiation respectively. The magnitude of $\mathrm{q}$ is given by $\mathrm{q}=2 \mathrm{k}_{0} \operatorname{Sin} \vartheta$ where $2 \vartheta$ is the angle of scattering (see Fig. 1), where $k_{0}=2 \pi / \lambda, \lambda$ being the wavelength of the incodent radiation. In general the small angle regime is defined by $\mathrm{q}<<\mathrm{k}_{0} . \mathrm{S}(\bar{q})$ in general is a function of the average of the instantaneous positions of all the particles in the scattering system, but in the small-angle regime (defined roughly by the range $0<\mathrm{q}<3 \mathrm{~nm}^{-1}$ ) we may ignore the atomic and molecular structure of the constituents and deal only with the spatial variations (on length scales from a few $\mathrm{nm}$ on up) of the scattering length density (SLD) $\rho(\vec{r})$ of the sample. For small-angle neutron scattering (SANS) experiments, $\rho(\vec{r})$ is defined by

$$
\rho(\overline{\mathrm{r}})=\Sigma b_{\mathrm{i}} n_{\mathrm{i}}(\overline{\mathrm{r}})
$$

where $b_{i}$ is the nuclear scattering length [5] of nucleus of type $I$, and $n_{i}(\vec{r})$ is the associated number density of such nuclei, while for small-angle X-ray scattering (SAXS) experiments, $\rho(\vec{r})$ is defined by

$$
\rho(\overline{\mathrm{r}})=\left(\mathrm{e}^{2} / \mathrm{mc}^{2}\right) \mathrm{n}_{\mathrm{el}}(\overline{\mathrm{r}})
$$

where the factor $\left(\mathrm{e}^{2} / \mathrm{mc}^{2}\right)$ is the Thompson scattering length of the electron, and $\mathrm{n}_{\mathrm{el}}(\overrightarrow{\mathrm{r}})$ is the electron number density. Since a uniform scattering length density does not scatter radiation (except in the forward direction), $S(\vec{q})$ will depend only on the deviations of $\rho(\bar{r})$ about its mean, or what is referred to as the contrast. The Kinematic or Born approximation to the scattering [6], where multiple scattering effects are neglected, then yields for the scattering function $S(\bar{q})$ the expression

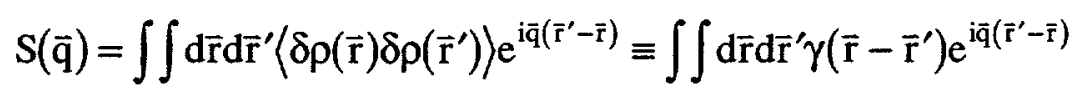

where the statistical average is taken over the whole system. (This statement has to be modified if the incident radiation is highly coherent. It is commonly assumed that such an average depends only on the magnitude of $\vec{R} \equiv \vec{r}-\vec{r}^{\prime}$, for an isotropic and translationally invariant system. In Eq. (5), $\delta p(\overrightarrow{\mathrm{r}})$ is defined as the fluctuation from the average SLD as explained above. If we are dealing with a particulate system (e.g., a dilute polymer solution) and these are far enough apart that the interference effects between the scattering from different particles (which will occur at values of $q$ typically of order $(2 \pi / d)$ where $d$ is the average interparticle distance) is not important in the range of q studied in the experiment, then Eq.(5) simplifies to 


$$
S(q)=(\Delta \rho)^{2} \sum_{i} v_{i}^{2} f_{i}^{2}(\bar{q})
$$

where the sum is over all particles, $v_{i}$ is the volume of the $i^{\text {th }}$ particle, $\Delta \rho$ is its SLD contrast with the average medium (assumed uniform throughout the particle and the same for all particles) and $f_{i}$ is its form factor defined by

$$
f_{i}(\vec{q})=\frac{1}{v_{i}} \int_{v_{i}} d \bar{r} e^{-i \bar{q} \cdot \vec{r}}
$$

where the integral is over the particle volume. In general, one can make a spherical average of $f_{i}^{2}(q)$ and assume some law of polydispersity in size of the particles to carry out the weighted sum in Eq.(6). In such a restrictive case (dilute system of random particles), by making an expansion for small $\overrightarrow{\mathrm{q}}$, one finds that

$$
S(q)=S(0)-\frac{1}{3} R_{g}^{2} q^{2}+\ldots
$$

where

$$
R^{2} g=R_{g}^{2} \sum\left(1 / v_{v_{i}}\right) \int_{v_{i}} d \vec{r} \cdot r^{2}
$$

is the average radius of gyration of the particles. Eq.(8) suggests (at least for small q) in this case the approximation

$$
S(q)=S(0) \exp \left(-\frac{1}{3} R_{g}^{2} q^{2}\right)
$$

which is the famous Guinier approximation [1] for scaling from a dilute system of uniform particles. If the particles are polymer chains, and the SLD or contrast for SANS experiments is modified by selective deuleration of either the chains or the solvent, the above considerations form the basis of the method for determining the radius of gyration of polymer chains is dilute solution.

A problem which has been studied by several groups by both SANS and light scattering techniques is that of the phase separation of a binary fluid mixture confined in a porous medium, such as Vycor glass or an aerogel [7-16]. A convenient system for such studies is a mixture of water and $(2,6)$ lutidine, which in bulk has an inverted phase diagram (with a homogeneous phase at low temperatures, and phase separation occurring at higher temperatures). The critical concentration in the bulk mixture is $31.2 \%$ lutidine, and the critical temperature is $33^{\circ} \mathrm{C}$. In the vicinity of the critical point in the single phase region, a bulk mixture shows critical fluctuations obeying 3D Ising-like behavior. The behavior in Vycor glass as seen by light scattering $[12,16]$ is very 
different and indicative of the effects of confinement and preferential wetting of the pores (typically $8 \mathrm{~nm}$ in diameter), with weak or non-existent critical fluctuations and long-time relaxation and hysteresis effects with temperature in the 2-phase region. SANS data from such a system [11,12] as a function of temperature is shown in Fig. 1. In order to study the concentration fluctuations in the fluid alone, without the complications from the scattering due to the solid/liquid contrast, the homogeneous phase was taken to be contrast-matched with the Vycor using the appropriate mixture of $\mathrm{H}_{2} \mathrm{O}, \mathrm{D}_{2} \mathrm{O}$ and lutidine. (The fact that Vycor preferentially absorbed lutidine from the supernatant solution complicated the task of ensuring that the final single-phase mixture inside the Vycor was nominally critical and contrast-matched, but this was achieved by a systematic study of different initial mixtures [11]). The "Vycor peak" at q 0.025 is absent due to the contrast- matching with the silica, but a peak at a larger value of $q$ $\left(\sim 0.035 \mathrm{~A}^{-1}\right)$ was observed in the data. This peak was identified as due to a "skin" of lutidine-rich liquid adsorbed on the internal pore surfaces, as proved by a complementary experiment, in which a similar layer of hydrocarbon chains was attached to the internal pore surface of Vycor inside a contrast-matching solution of H/D toluene in the pores. The peak at the same q was clearly observed (see Fig. 2) and was used to subtract off the "skin" scattering from the observed S(q) from the Vycor/water/lutidine mixture i.e. from the observed data in Fig. 1. The remaining scattering was fitted by the sum of a Lorentzian to represent the critical fluctuations (which turned out to have a very small amplitude) and a Lorentzian-squared term (with a different length-scale) to represent the formation of microdomains of phase-separated water-rich and lutidine-rich phases in the 2-phase region). The fitted curves are shown in Fig. 1. A good fit was obtained, with the domain size saturating in the 2-phase region at the $8 \mathrm{~nm}$ length-scale of the Vycor pores. This provided partial confirmation of the phase diagram obtained theoretically by Liu et al. [15] for a fluid mixture phase separating inside a finite tube. In their phase diagram (see Fig. 3), the system goes from a "tube" phase (lutidine-rich "skin" lining the pore walls) to a "capsule"-like phase ("skin" and capsules of water-rich phase within the tubes) as the temperature is raised into the 2-phase region.

A similar study was carried out by Frisken et al. [14] in the much more open structures of a series of silica gels using a $\mathrm{D}_{2} \mathrm{O}$ lutidine mixture. In this case, the scattering was interpreted as that from a dispersed fractal structure of the silica framework, together with the associated static concentration variations induced in the fluid by the preference of the silica for wetting itself with lutidine, in addition to spontaneous critical fluctuations in the fluid. Thus this model is similar in spirit to the well-known "Random Field Ising Model" which was initially proposed for such systems $[7,8]$. 


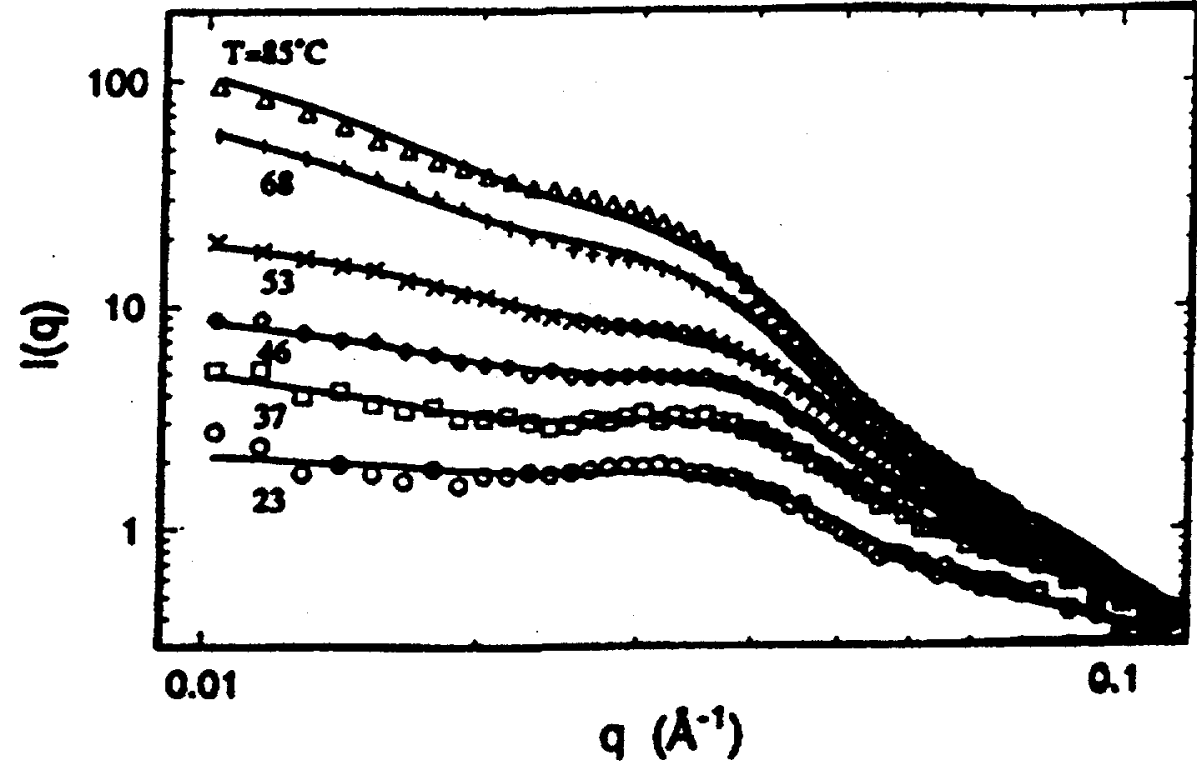

Figure 1. S(q) (arbitrary units) for a contrast-matching binary lutidine/water mixture near the critical concentration inside Vycor glass, as a function of temperature. (From Ref. [11]).

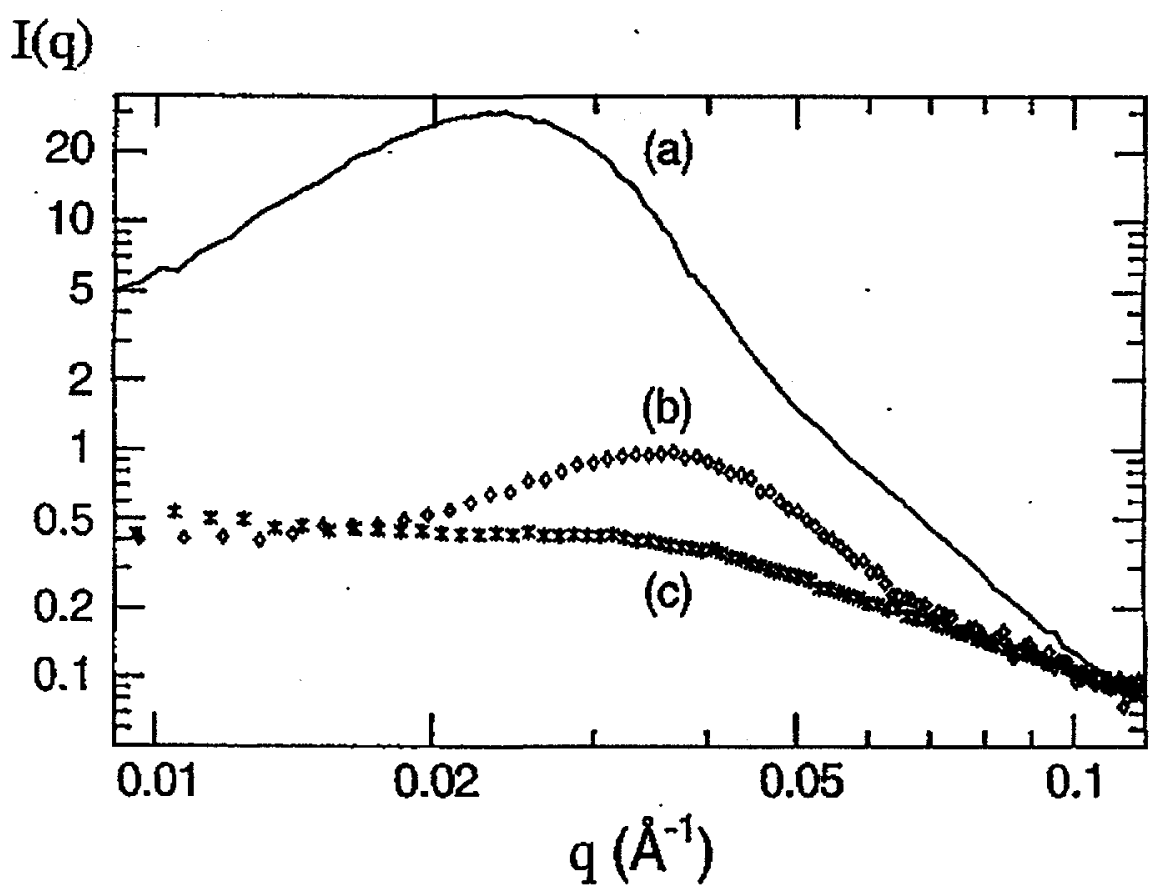

Figure 2. $S(q)$ (log-log plot, arbitrary units) for (a) dry Vycor glass (b) Vycor derivalized with $\mathrm{C}_{18^{-}}$ alkylsiloxane surface layer and filled with contrast-matching hexane/d-hexane mixture and (c) Vycor with contrast-matching binary lutidine/water mixture. (From Ref. [11]). 


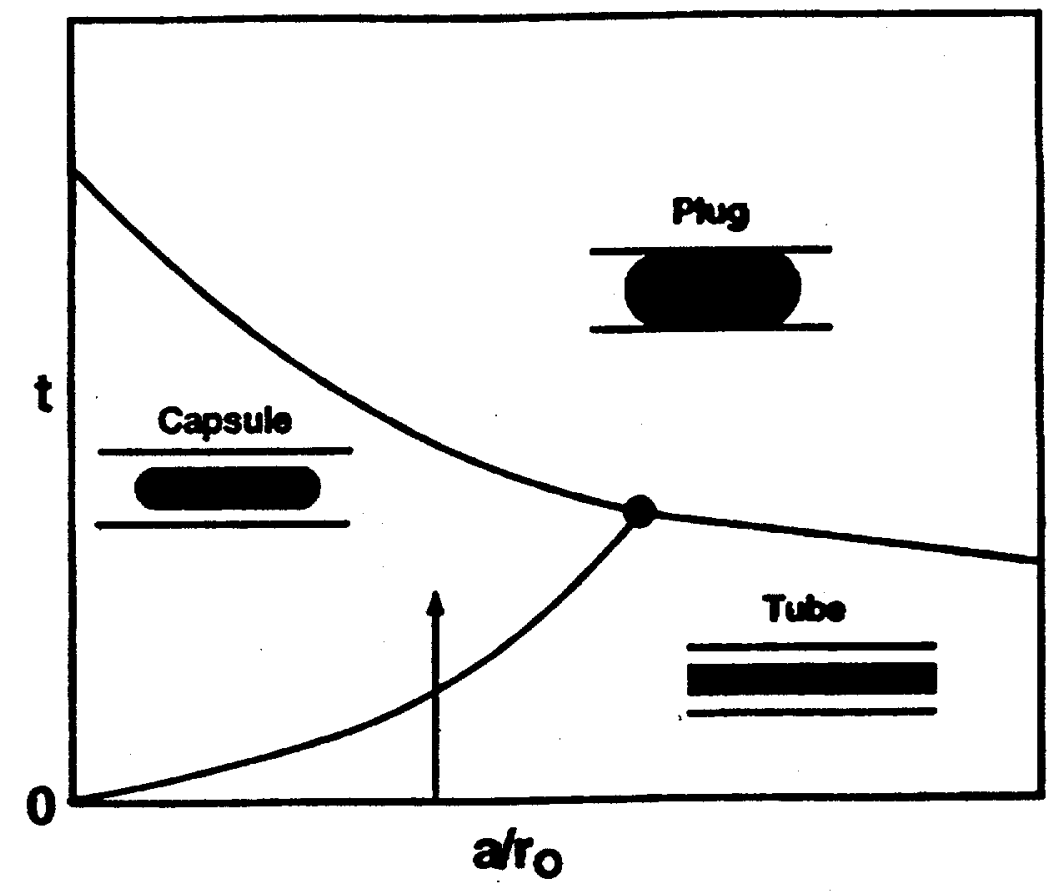

Figure 3. Phase diagram of the single-pore model of Liu, et al (Ref. [15]) for phase separation inside a confined pore geometry. The arrow indicates the observed behavior of the water/lutidine system in Vycor deduced from SANS data. $t$ is the reduced temperature, $r_{0}$ is the tube radius and $a$ is a molecular length.

Following Eq.(5) we may write

$$
S(q)=\frac{1}{V}\left\langle\delta \rho(\vec{q}) \delta \rho^{*}(\vec{q})\right\rangle
$$

where $\delta \rho(\vec{q})$ is the Fourier transform of the SLD fluctuation $\delta \rho(\vec{r})$. If $\phi_{s}(r), \phi_{1}(r), \phi_{w}(r)$ are respectively the local volume fractions of silica, lutidine and $\mathrm{D}_{2} \mathrm{O}$ and their fluctuations from the mean are $\delta \phi_{s}, \delta \phi_{1}, \delta \phi_{w}$, then

$$
\rho(\vec{q})=\rho_{s} \phi_{s}(\vec{q})+\rho_{1} \phi_{1}(\vec{q})+\rho_{w} \phi_{w}(\vec{q})
$$

where $\rho_{s}, \rho_{1}, \rho_{w}$ are the SLD's of silica, lutidine and water respectively. Since

$$
\delta \phi_{s}+\delta \phi_{1}+\delta \phi_{w}=0
$$


We obtain from Eq. (12)

$$
\delta \rho(\vec{q})=\left(\rho_{s}-\rho_{w}\right) \delta \phi_{s}(\bar{q})+\left(\rho_{1}-\rho_{w}\right) \delta \phi_{1}(\bar{q})
$$

Let us ignore spontaneous critical concentration fluctuations in the liquid for the moment and concentrate on the response of the lutidine concentration to the silica concentration via the "wetting" interaction. Assuming linear response, this may formally be written as

$$
\delta \phi_{1}(\overline{\mathrm{q}})=\alpha(\overrightarrow{\mathrm{q}}, \mathrm{T}) \delta \phi_{\mathrm{s}}(\overrightarrow{\mathrm{q}})
$$

where $\alpha(\vec{q}, T)$ may be written in the Ornstein-Zernike form

$$
\alpha(\bar{q}, T)=-\frac{\bar{\phi}_{1}}{\bar{\phi}_{1}+\bar{\phi}_{w}}+\frac{\alpha_{0}(T)}{1+q^{2} \xi^{2}}
$$

(The constant term in Eq. (16) is there to account for the excluded volume decrease of lutidine concentration due to the presence of the silica, even in the absence of wetting, i.e., when $\alpha_{0}=\mathrm{O}$ ).

Using Eqs. (14-16) in Eq. (11), we obtain

$$
S(q)=\frac{1}{V}\left[\left(\rho_{s}-\rho_{w}\right)+\left(\rho_{1}-\rho_{w}\right) \alpha(\vec{q}, T)\right]^{2}\left\langle\delta \phi_{s}(\vec{q}) \delta \phi_{s}^{*}(\vec{q})\right\rangle
$$

But the scattering from the silica gel itself in pure $\mathrm{D}_{2} \mathrm{O}$ may be written as

$$
S_{s g}(q)=\frac{1}{V}\left[\rho_{s}-\rho_{w}\right]^{2}\left\langle\delta \phi_{s}(\bar{q}) \delta \phi_{s}^{*}(\bar{q})\right\rangle
$$

so that the scattering from the binary fluid mixture may be expressed in terms of $\mathrm{S}_{\mathrm{sg}}(\mathrm{q})$ (measured in a separate experiment) and $\alpha(\overrightarrow{\mathrm{q}}, \mathrm{T})$ as

$$
S(\bar{q})=\left[1+\frac{\rho_{1}-\rho_{w}}{\rho_{s}-\rho_{w}} \alpha(\bar{q}, T)\right]^{2} S_{s g}(\bar{q})
$$

To this must be added the pure critical fluctuations in the fluid given by

$$
S_{\text {crit }}(\bar{q})=\left(\rho_{1}-\rho_{w}\right)^{2} \chi \prime\left(1+q^{2} \xi^{2}\right)
$$


and a background term. Such an expression was found to provide an extremely good fit (Fig. 4) to the data for a wide range of temperatures and concentrations throughout the one-phase region of the pure system. The four fitting parameters used were $\chi, \xi, \alpha_{0}$ and a constant background term B.

The results showed that $\xi$ increased towards the critical temperature, as did $\alpha_{o}$ and $\chi$, although the accuracy was not sufficient to determine any critical exponents (in addition to the fact that the concentration of the "free" fluid, that which is not "frozen" in the wetting layers, is also changing with temperature). This behavior appears to be different to the behavior observed in Vycor. $\alpha_{0}$ was also found not to scale with $\chi$, which would be expected from a simple linear response theory indicating that the wetting response of the fluid near the silica surface is probably non-linear as might be expected. In the 2 -phase region, $S(\bar{q})$ could be represented by

$$
S(q)=\frac{C_{1}}{\left(1+q^{2} \xi_{d}^{2}\right)^{2}}+C_{2} S_{s g}(q)
$$
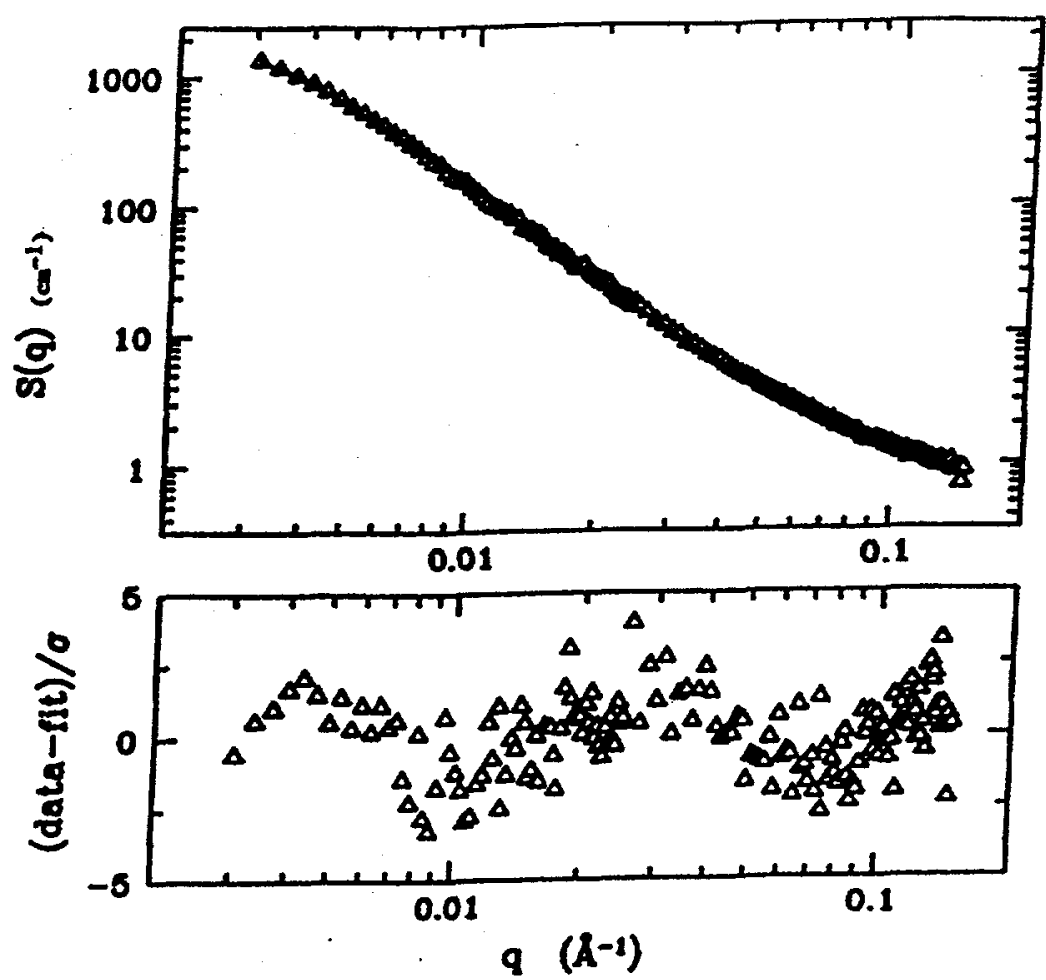

Figure 4. Result of fitting the theoretical expression given in Eqs. (18) and (19) to the measured $S$ (q) (in absolute units) for a silica gel/water/lutidine sample containing $2.05 \%$ silica and 40 volume of lutidine at $26.80^{\circ} \mathrm{C}$. The residuals in units of the standard deviation of the data are shown in the bottom portion of the figure. (From Ref. [14]) 
plus a constant background term, i.e., scattering due to the silica gel in an effective uniform fluid plus scattering from domains indicating that sharp interfaces between phase separated domains had formed. Eq. (21) is similar in spirit to the model of Debye et al. [17], and the models used by Wong et al. [18] and Lin et al. [11] to describe frozen domains. $\xi_{\mathrm{d}}$ was too large to measure accurately. Within the instrumental resolution, the first term in Eq. (21) effectively looked like Porod scattering. These domains also appeared to coarsen with increasing time, as observed by an increase in the amplitude of the $\mathrm{q}^{-4}$ term (proportional to the interfacial area by Porod's Law).

The conformation of polymers confined inside pore spaces is also of interest. At first thought it would appear difficult to get a polymer whose equilibrium radius of gyration $\mathbf{R}_{\mathrm{g}}$ in solution is greater than a typical pore size to enter the porous medium. However, it turns out, as predicted theoretically [19] that from a sufficiently concentrated (semi-dilute or more concentrated) polymer solution osmotic pressure will force the polymer chains to enter the pores, the criterion being roughly that the correlation length $\xi$ in the semi-dilute solution be comparable to the pore size. This was observed in SANS experiments by Lal et al [20] where polystyrene (PS) chains of equilibrium radius of gyration as large as $30 \mathrm{~nm}$ were imbibed into cleaned Vycor glass from semi-dilute solutions in times ranging from hours (for the smallest chains) to 65 days (for the largest chains). This was verified by first studying the SANS from the virgin Vycor sample with a contrast-matched solvent (deuterated dichloromethane) in the pores (which showed no characteristic "Vycor peak") and comparing it with the imbibed Vycor in which the Vycor peak had reappeared, indicating that PS chains entering the Vycor had destroyed the contrast-matching condition.

For the SANS experiments a mixture of hydrogenated and deuterated PS chains of equal molecular weights (MW) (h-PS and d-PS respectively), and hydrogenated and deuterated toluene solvent (h-toluene and d-toluene) was used. The SANS experiment can be made sensitive to the conformation of individual PS chains inside the Vycor if the following two conditions are simultaneously met:

1) The h-, d-toluene mixture exactly contrast matches the silica of the Vycor. (This was determined by careful subsidiary contrast matching experiments using varying $h-$, and dtoluene concentrations.)

2) The concentration of h-PS and d-PS chains is chosen such that the averaged SLD of the chains exactly matches that of the solvent.

Under these conditions it may be shown [20] that only single chain fluctuations will contribute to the observed $S(q)$ which thus measures the form-factor or conformation of simple chains. Experiments were done for a variety of PS molecular weights. A typical S(q) curve for PS of MW 75K in toluene in Vycor is shown in Fig. 5. The results were compared with the $S(q)$ for the same chains in dilute solution in toluene (i.e. "free" chains). A Debye-function fit (appropriate to free Gaussian chains in a good solvent) [20] was made to both sets of data. Such a procedure is valid for obtaining a radius of gyration from the small $\mathrm{q}$ region where the fit is reasonably good, although at large $\mathrm{q}$ both curves deviate. The radius of gyration of the confined chains were obtained 


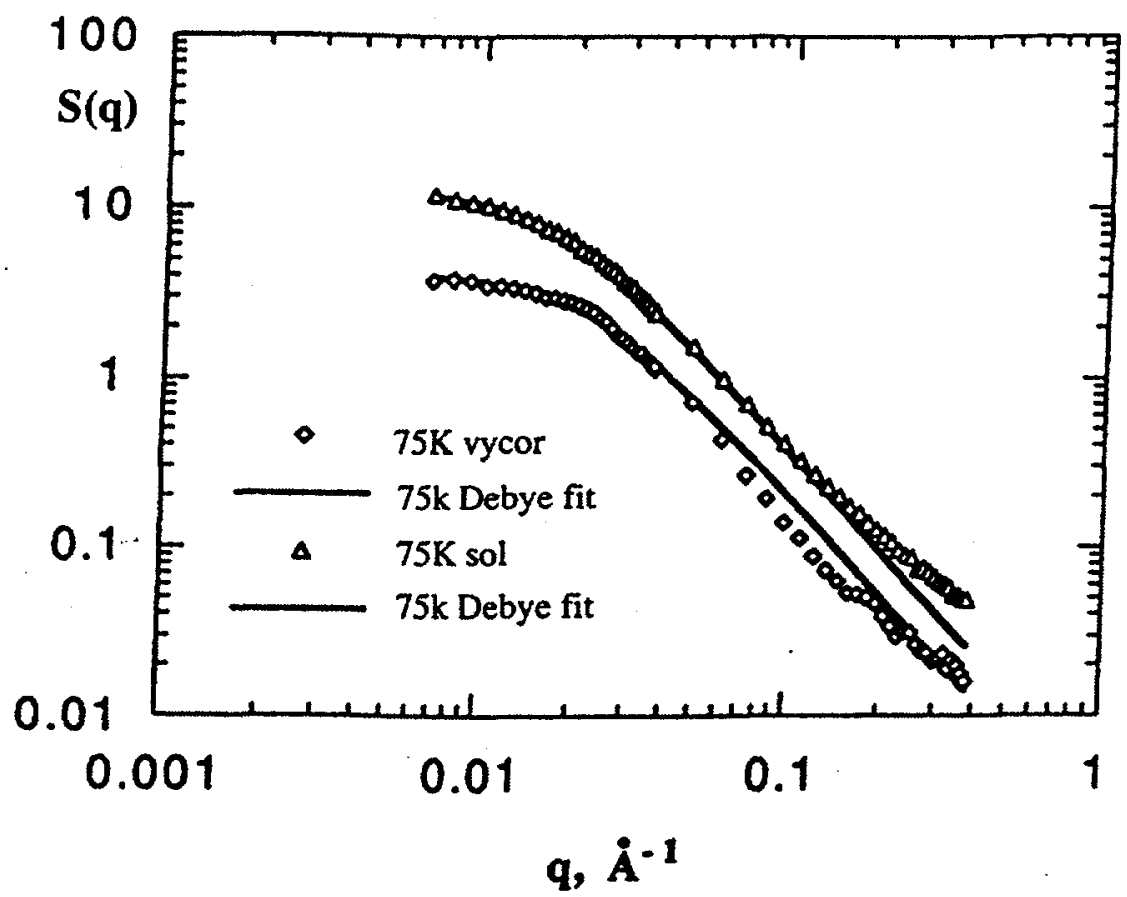

Figure 5. S(q) (arbitrary units) for $75 \mathrm{~K} \mathrm{MW}$ polystyrene chains in dilute solution $(\Delta)$ in toluene and in toluene in Vycor $(0)$. The solid lines are corresponding fits of the Debye-function for ideal polymer chains. (From Ref. [20])

to be always smaller than the radius of gyration of the free chains in the equivalent bulk solution. The free chains in dilute solution yield an $S(q)$ that deviates from the $1 / q^{2}$ Debye-like behavior due to excluded volume effects (yielding $q^{-1 / v}$, where $v$ is the Flory exponent [21]). The chains in the Vycor deviate due to conformational changes arising from confinement in the tube-like pores. These were interpreted in terms of the theory developed by Daoud and de Gennes [22] and Brochard and de Gennes [23]. Briefly, the theory takes into account four main effects which govern the behavior of chains confined in non-adsorbing cylindrical pores. These are the confinement of the chains by the cylinder walls, which squeezes the chains laterally, the intrachain excluded volume effect which swells the chains and stretches them along the cylinder axis, the entropic elasticity of the chains which limits this stretching, and the interchain interactions which may also lead to segregation of the chains. Thus the polymer chains are stretched out into "cigars" along the tubes, which may at high concentrations segregate from each other (strong confinement limit) or overlap in an entangled manner (moderate confinement regime) and thus no longer be stretched along the cylinder axis. In the latter cases $R_{g z}^{2}=R_{g}^{2} / 3$, where $R_{g z}$ is the radius of gyration along the cylinder axis, and $R_{g}$ is that of the chain in bulk solution. One then has the relation

$$
\mathrm{R}_{\mathrm{gv}}^{2}=\mathrm{D}^{2} / 8+\mathrm{R}_{\mathrm{gz}}^{2}
$$


where $R_{\mathrm{gv}}$ is the radius of gyration as measured in the Vycor pore space and $\mathrm{D}$ is the pore diameter of Vycor (known to be $\sim 7 \mathrm{~nm}$ ). Thus Eq.(22) provides a method for testing the relationship of the measured radii of gyration of the individual chains in Vycor and in the bulk solution, and was found to be satisfied extremely well for the different molecular weights studied. Thus one can conclude that the chains were in the conformation of ideal overlapping squeezed cigars. This conclusion was confirmed by also fitting the $S(q)$ of the individual chains in the regime $D<q^{-1}<R_{g}$ to a "cylindrical" Guinier model.

\section{Studies of Fluids at Interfaces and in Thin Films}

At a liquid/vapor or liquid/solid interface, the liquid behavior may be modified in several ways. Near the melting temperature, the bulk may be crystalline in coexistence with a thin layer of liquid at the interface (surface melting) or the liquid phase may develop as crystalline layer at the interface (surface or interface freezing). There have been a fair number of studies of such phenomena over the last few years. Here, we shall discuss studies of this phenomenon on hydrocarbon liquid systems as probed by X-ray reflectivity.

Specular X-ray reflectivity yields the electron density profile normal to the liquid surface (averaged over the plane of the surface), and thus can reveal the existence of a surface layer with a slightly different density to that of the bulk. In the simplest theory, the specular reflectivity is given by the expression [24]

$$
R=R_{F}\left|\frac{1}{\rho_{o}} \int_{-\infty}^{\infty} d z \frac{d \rho(z)}{d z} e^{i q_{z} z}\right| 2
$$

where $q_{z}$ is the wavevector transfer normal to the surface.

(This expression breaks down when the reflectivity approaches unity in the vicinity of total external reflection, at small values of $\mathrm{q}_{\mathrm{z}}$. In this region, there are standard methods, borrowed from optics, to calculate the reflectivity accurately by regarding the varying electron density normal to the surface as made up of a series of thin slabs with constant density, matching boundary conditions and using iterative procedures to solve for the fields $[25,26])$.

In the isotropic phase of a liquid crystal, the free surface can produce an alignment to a nematic phase which penetrates further into the bulk as the temperature is lowered towards the bulk isotropic-nematic transition temperature $\left(\mathrm{T}_{\mathrm{IN}}\right)$ [27]. We may say that the nematic phase wets the surface slightly above $T_{\mathbb{I N}_{\mathrm{N}}}$. Similarly, the smectic phase can begin to wet the surface in the nematic phase as $T$ tends to the nematic-smectic transition temperature and this effect has been studied by $\mathrm{X}$-ray specular reflectivity techniques by 
Pershan, Als-Nielsen and co-workers [28], using a liquid surface reflectometer. Fig. 6 shows the measured specular reflectivity from the surface of the liquid crystals Octyloxycyanobiphenyl (8OCB) as the temperature is decreased towards the nematicsmectic A transition temperature $\mathrm{T}_{\mathrm{NA}}$. Plotted are both $R\left(q_{z}\right)$ and $R\left(q_{z}\right) / R_{F}\left(q_{z}\right)$ where $R_{F}$ is the theoretical Fresnel reflectivity. For $q_{z} \ll Q_{o}$ (where $Q_{o}$ is $2 \pi$ divided by the periodicity of the smectic layers), $R\left(q_{z}\right)$ follows the Fresnel reflectivity $R_{F}\left(q_{z}\right)$ fairly

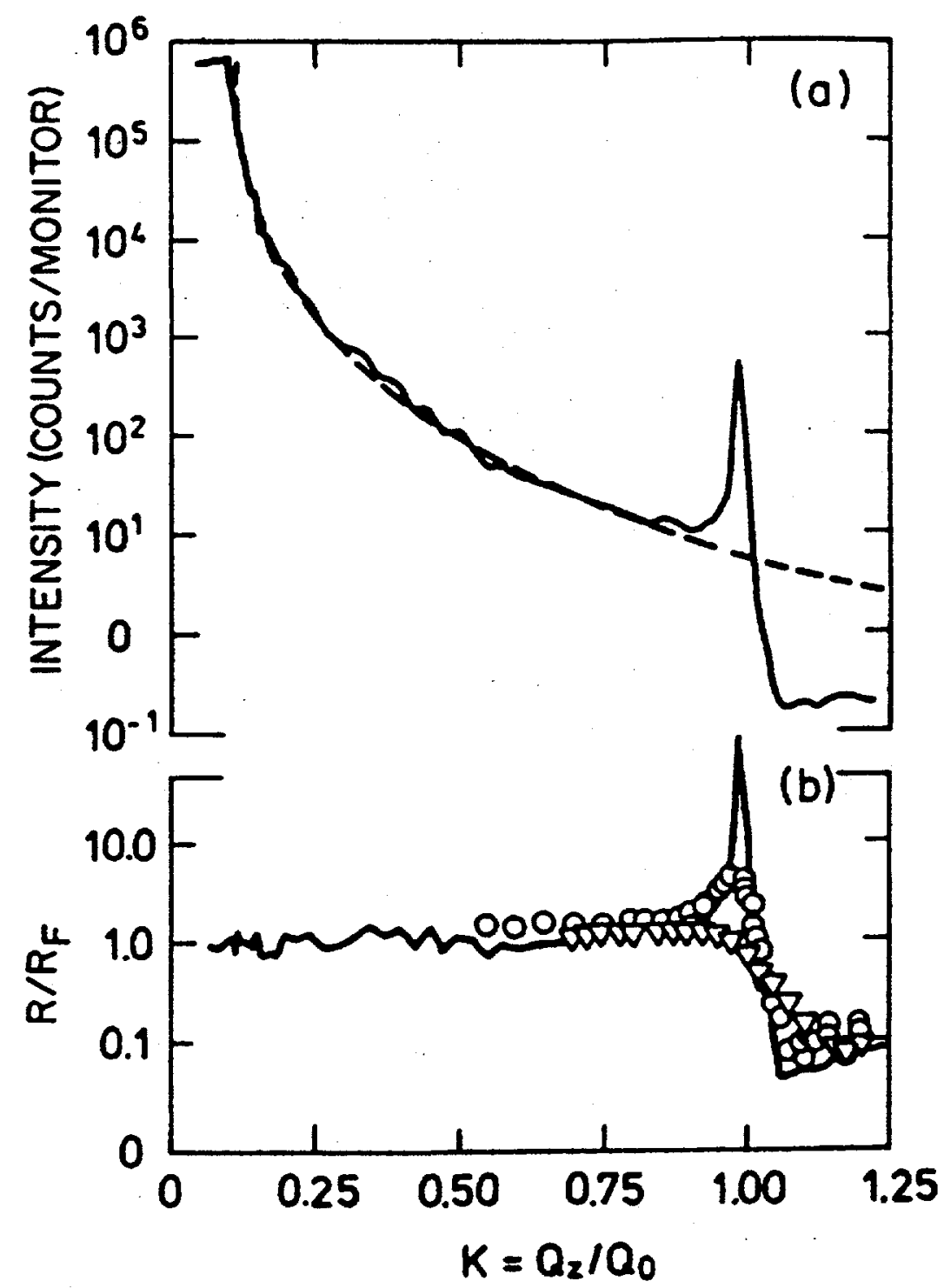

Figure 6. (a) Specular $x$-ray reflectivity from the surface of $80 C B$ at $T-T_{N A}=0.05^{\circ} \mathrm{C}$. The $Q_{z}$ scale is normalized to $Q_{0}=0.199 \AA^{-1}$. The dashed line is the calculated Fresnel reflectivity (b) $R\left(Q_{2}\right) / R_{F}\left(Q_{2}\right)$ vs $\mathrm{Q}_{z} / \mathrm{Q}_{11}$. The solid line is for $\mathrm{T}-\mathrm{T}_{\mathrm{NA}}=0.05^{\circ} \mathrm{C}$, open circles for $\mathrm{T}-\mathrm{T}_{\mathrm{NA}}=2.8^{\circ} \mathrm{C}$ and the triangles for $\mathrm{T}$ $\mathrm{T}_{\mathrm{NA}}=11.06^{\circ} \mathrm{C}$. (after Pershan et al., Ref. [28]) 
well. For $q_{z} \sim Q_{o}$, the smectic peak appears with a width proportional to $\xi_{\text {, }}$, the correlation length in the direction normal to the smectic layers. This peak sharpens as $\mathrm{T}_{\mathrm{NA}}$ is approached. For $q_{z} \geq Q_{o}, R\left(q_{z}\right)$ dips sharply below $R_{F}\left(q_{z}\right)$. This is due to destructive interference between the surface reflectivity and that from the smectic layers. To fit the data, the authors used the formula given in Eq. (23) with

$$
\rho(z)=\rho_{0}(z)+\rho_{1}(z)
$$

where the first term was taken to be given by

$$
\frac{1}{\rho_{\infty}} \frac{d \rho_{0}}{d z}=\frac{d}{d z} \Theta\left(z-z_{o}\right) B_{s} \exp \left[-\frac{z-z_{o}}{\xi_{\uparrow}(T)}\right] \operatorname{Sin}\left[Q_{o}\left(z-z_{o}\right)\right]
$$

( $\Theta\left(z-z_{o}\right)$ being the step-function which is 1 if $z>z_{o}$, and 0 otherwise) and the second term was taken to be

$$
\frac{1}{\rho_{\infty}} \frac{d \rho_{1}}{d z}=C_{1}(\pi z)^{-1} \exp \left[\frac{-(\sigma z)^{2}}{2}\right] \operatorname{Sin}\left(Q_{1} z\right)
$$

where $C_{1}$ is defined to ensure $\int \rho_{1}^{-1} d \rho_{1} / d z=1$.

The Fourier transforms then yields

$$
R\left(q_{z}\right)=R_{F}\left(q_{z}\right)\left|\Phi\left(q_{z}\right)\right|^{2}
$$

where

$$
\Phi\left(q_{z}\right)=\Phi_{0}\left(q_{z}\right)+\Phi_{1}\left(q_{z}\right)
$$

and

$$
\begin{gathered}
\Phi_{0}\left(q_{z}\right)=i \frac{B_{s}}{2} \exp \left(-i q_{z} z_{0}\right)\left[\frac{\xi_{1} Q_{o}-1}{\left(q_{z}-Q_{o}\right) \xi_{\|}-i}-\frac{\xi_{1} Q_{o}+1}{\left(q_{z}+Q_{o}\right) \xi_{\mid}+i}\right] \\
\Phi_{1}\left(q_{z}\right)=\left(\frac{C_{1}}{2}\right)\left[\operatorname{erf}\left[\frac{q_{z}+Q_{1}}{\sqrt{2} \sigma}\right]-\operatorname{erf}\left[\frac{q_{z}-Q_{1}}{\sqrt{2} \sigma}\right]\right]
\end{gathered}
$$

The term $\rho_{0}$ represents a sinusoidal (smectic) density decaying into the bulk with correlation length $\xi_{\|}$, with a phase which is controlled by $z_{0}$, while $\rho_{1}$ represents a 
surface smeared with a roughness $\sigma$ but with also a sinusoidally varying component which is damped rapidly into the bulk. It is basically an empirical from chosen to represent the experimental data, and is presumed to correct for the fact that the form of Eq. (25) for $\rho_{0}$ does not accurately represent the smectic oscillations near the surface. Fitting of the data using Eq. (27) yields a value for $\xi_{\|}$essentially identical to the bulk correlation length measured in X-ray scattering experiments on bulk samples in the region near the phase transition. It also yields a value for $z_{0}$ which is $0.25 \mathrm{~d}$, $\mathrm{d}$ being the layer spacing of the smectic-A phase $\left(=2 \pi / Q_{o}\right)$, yielding a maximum in $\rho_{0}$ exactly $\mathrm{d} / 2$ away from the surface. $Q_{1}$ turned out to be equal to $Q_{0}$ within a few percent. An interesting result of this experiment was the fact that the specular peak at $q_{z}=Q_{o}$ was extremely sharp in the transverse direction, while the bulk critical scattering showed a finite transverse correlation length $\xi_{\perp}$. Thus the smectic order at the surface had a much larger in-plane correlation than in the bulk.

In another experiment on the system dodecylcyanobiphenyl (12CB) which does not have a smectic phase, but a first-order transition from the bulk isotropic phase to the smectic-A phase at $\mathrm{T}_{\mathrm{IA}}=57.7^{\circ} \mathrm{C}$, Ocko et al. [29] found a specular reflectivity that clearly showed interference effects developing due to smectic layers forming at the surface above $T_{I A}$ as the transition temperature was approached. The data showed quantization effects as a function of temperature due to discrete smectic layers forming. Up to 5 smectic layers were observed as the temperature was lowered. The data was again fitted with expression (23) with the form taken for $\rho(z)$ being given by

$$
\rho(z)=\Theta\left(z-z_{o}\right)+H_{n}(z) B_{s} \operatorname{Sin}(2 \pi z / d)
$$

where $\Theta(z)$ is the step-function defined in Eq. (25), d the smectic layer spacing and $H_{n}(z)=1$ for $0<\mathrm{z}<$ nd and zero elsewhere. The above expression was convoluted with a Gaussian profile to represent surface or interlayer roughness and the resulting reflectivity fitted to the experimental data by adjusting $z_{0}, B_{s}$ and the roughness parameter. The results showed an (incomplete) wetting of the free surface of the isotropic phase of $12 \mathrm{CB}$ by the smectic-A phase, but in discrete layers, rather than with an exponential decay into the bulk. Ocko has also used X-ray specular reflectivity experiments to demonstrate such layering transitions at smectic A-solid interfaces [30].

In view of the fact that the free surface has a disordering effect on a solid, and that surface melting appears to be common in ordinary solids [31], the ordering of liquid crystals at the free surface is somewhat unexpected. Holyst [32] has proposed a theory which may account for the layer-by-layer freezing in terms of the quenching of the outof-plane surface fluctuations by surface tension effects.

Liquid crystals are not the only systems to show surface ordering effects. Even the simplest chain-like hydrocarbon molecules, namely the normal alkanes (which possess no nematic or smectic phases) show such behavior. Fig. 7 shows the specular X-ray 


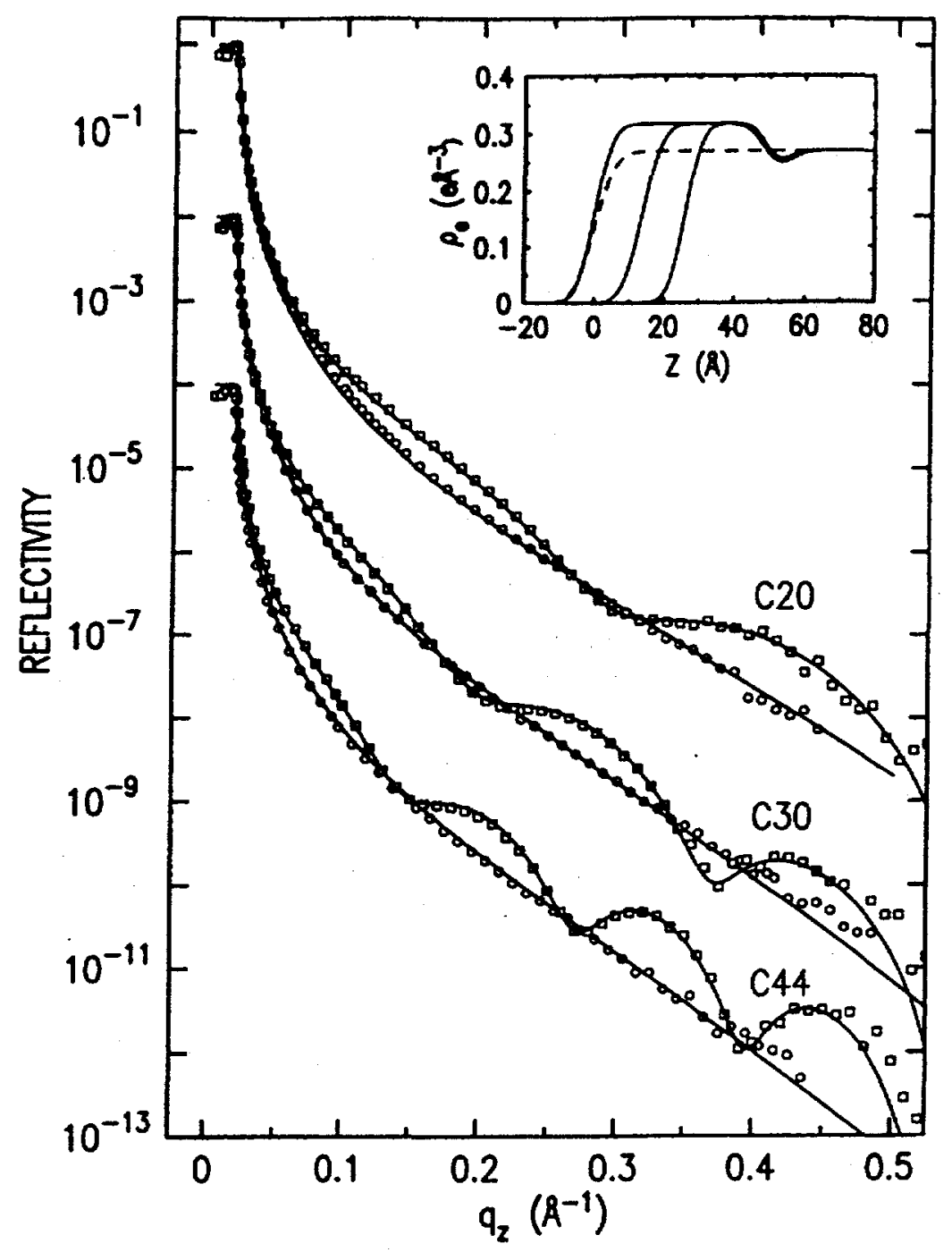

Figure.7. Specular $x$-ray reflectivity data for liquid alkanes with carbon numbers $n=20,30$, and 44 respectively at $T_{m}+4^{\circ} \mathrm{C}$ (open circles) and $T_{m}+3^{\circ} \mathrm{C}$ (open squares), where $T_{m}$ is the bulk freezing temperature for each. The inset shows model fits in the crystalline (solid) and liqid (dashed) surface phases of the electron densities at the interfaces. (From Ref. [33])

reflectivity from the surface of 3 liquid alkanes (carbon numbers $=18,20,24$ respectively) at roughly $4^{0} \mathrm{C}$ above their respective bulk crystallization temperatures $\mathrm{T}_{\mathrm{b}}$. [33]. At the higher temperature, the reflectivity is well fitted by the simple Nevot-Croce form as expected for a simple liquid surface with an effective roughness due to capillary wave fluctuations. However at about $3^{\circ} \mathrm{C}$ above $\mathrm{T}_{b}$, oscillations in the reflectivity indicate the presence of a slightly denser layer on the liquid surface, with a thickness approximately equal to the chain length of the molecule. (The density profile fitted to the reflectivity for the C24 chain in this regime is shown in the inset to Fig. 7). The 
electron density of this layer is close to that of the bulk "rotator" crystalline phase, where the chains are oriented in a hexagonal structure normal to the layers, but the bonds along the chain are disordered. These reflectivity experiments thus suggest strongly that a single layer of this crystalline phase has formed on the surface of the bulk liquid slightly above $T_{b}$. This was confirmed by in-plane Grazing Incidence X-ray Diffraction (GIXD) experiments, and also by surface tension measurements [34]. Unlike the 12CB liquid crystal experiment discussed above, only one layer of the crystalline phase was seen to form before the bulk phase was reached via a strongly first order transition. Subsequent reflectivity and GIXD experiments showed that for long chain alcohols, a similar surface crystalline phase also formed slightly above $\mathrm{T}_{\mathrm{b}}$. This surface phase, however, consisted of a single bilayer of the hexagonally close-packed and tilted alcohol chains [35]. Reflectivity experiments have also shown surface crystallization in mixtures of alkane chains of different lengths [36]. For mixtures of chains of fairly comparable lengths, the thickness of the layer yields an average of the chain-length of the two species. For fairly disparate chain length mixtures on the other hand, one observes surface crystallization of either the majority component only or no surface crystallization at all when the concentrations are nearly equal. This has been explained [36] as being due to the extra energy cost of cocrystallizing chains of very different length adjacent to each other. GIXD experiments show that in these monolayer surface crystalline phases, there also exist tilted phases, i.e. where the alkane or alcohol molecules, instead of being oriented normal to the surface, develop a tilt angle towards nearest-neighbor or next-nearest-neighbor positions.

There have been measurements, testing the surface force apparatus (SFA) which indicate that many non-liquid-crystal fluids confined in thin films between two solid surfaces show ordering in layers parallel to the surface, not seen in the bulk, as evidenced by oscillatory forces between the surfaces as a function of separation [37]. (Oscillatory order of a bulk fluid in the vicinity of an interface with a solid has also recently been observed by X-ray reflectivity [38]). If the film is only a few molecular diameters thick, this layered structure exists across the film, and in addition the film may develop lateral order (epitaxially with the wall surfaces, or otherwise) forming a crystalline phase induced by confinement, as evidenced by computer simulations [39], and SFA experiments involving shear between the surfaces showing stick-slip behavior [40]. Obviously, apart from being of fundamental interest, such ordering effects are important in understanding lubrication or adhesion involving fluid films between solid surfaces.

We turn now to studies of fluctuations at liquid surfaces. The scattering may be calculated in terms of the height-height correlation function $\langle\delta z(\vec{r}) \delta z(\vec{r}+\vec{R})\rangle$ where $\delta z(\vec{r})$ is the vertical height fluctuation (along the $z$ axis normal to the surface) at lateral position $(\vec{r})$, and $(\vec{R})$ is the lateral separation between the two points. If we denote this function by $C(\vec{R})$, then the scattering cross section as a function of $\bar{q}$ is given by 


$$
S_{\text {diff }}(\vec{q})=A \frac{(\Delta p)^{2}}{q_{z}^{2}} \iint d X d Y\left[e^{q_{z}^{2} C(R)}-1\right] e^{-i\left(q_{x} X+q_{y} Y\right)}
$$

where $(\Delta \rho)$ is $\left(\mathrm{e}^{2} / \mathrm{mc}^{2}\right)$ times the electron density difference across the liquid surface, and $A$ is the surface area. We have excluded the specular component of this expression, so that the above expression describes the diffuse or off-specular component of the scattering.

In the case of liquid surfaces, surface roughness is due to capillary wave fluctuations. There are some problems in connection with a truly first-principle calculation of such fluctuations (for a recent discussion, see Ref. 41), but one may write down at least a phenomenological expression for the surface free energy of the liquid and derive from it the spectral function for surface height fluctuations in the form:

$$
\left\langle\left.\delta z(q)\right|^{2}\right\rangle=\frac{k T}{\gamma\left(q^{2}+k^{2}\right)}
$$

where $\gamma$ is the surface (or interface) tension, and $\kappa$ is the inverse of the capillary length defined by $\kappa^{-1}=\left(\Delta \rho_{0}\right) g / \gamma, \Delta \rho_{0}$ being the mass-density difference between the fluids on either side of the interface. For bulk liquids $\kappa$ is typically of the order of $10 \mathrm{~cm}^{-1}$. Fourier transformation of Eq. (33) leads to a form for the height-height correlation function

where

$$
C(r)=-\frac{1}{2} B K_{0}(K r)
$$

$$
\mathrm{B}=\mathrm{kT} /(\pi \gamma)
$$

and $\mathrm{K}_{0}(\mathrm{x})$ is the modified Bessel function. At length scales $<<\mathrm{K}^{-1}$ (which are, in practice, those relevant for scattering experiments), the Bessel function may be replaced by a logarithm, and, to prevent short (molecular) length scale problems, we may also introduce a lower length scale cutoff [42]. Thus, we finally write

$$
C(r)=-\frac{1}{2} B \ln \left[\kappa\left(r^{2}+r_{o}^{2}\right)^{\frac{1}{2}}\right]
$$

where $r_{o}$ is defined to give the correct lateral surface roughness given by the integral of Eq. (33) ( $r_{o}$ turns out to be the inverse of the upper q cutoff for the capillary waves, $q_{u}$ defined below).

From Eq. (33) we see by integration over $\mathrm{q}$ that the true mean square roughness due to surface capillary waves is given by 


$$
\sigma^{2}=\frac{1}{4} B \ln \left[\left(q_{u}^{2}+\kappa^{2}\right) / \kappa^{2}\right]+\sigma_{o}^{2}
$$

where $\sigma_{0}$ is an "intrinsic roughness" due to the size of the molecules at the surface, and $\mathrm{q}_{\mathrm{u}}$ is an upper cut-off for the capillary wavevectors introduced to make the integral converge. It is $\left(1 / r_{o}\right)$ where $r_{o}$ is the cut-off introduced in Eq. (36). Since $\kappa$ is in general $\ll \mathrm{q}_{\mathrm{u}}$, Eq (37) may be written as

$$
\sigma^{2}=\frac{1}{2} \mathrm{~B} \ln \left(\mathrm{q}_{\mathrm{u}} / \kappa\right)+\sigma_{\mathrm{o}}^{2}
$$

Substituting this in Eq. (32), we may calculate the scattering after folding with the resolution function. If the latter is approximated by a Gaussian, Sanyal et al [43], have derived the form for the scattered intensity at $q_{x}, q_{z}$ (with $q_{y}$ integrated over assuming wide slits out of the plane of scattering)

$$
I=I_{0} \frac{q_{c}^{4}}{16} \frac{1}{q_{z}^{3}}\left(\frac{1}{2 k_{o} \operatorname{Sin} \alpha}\right) \exp \left(-q_{z}^{2} \sigma_{\text {eff }}^{2}\right) \frac{1}{\sqrt{\pi}} \Gamma\left[\frac{1-\eta}{2}\right]_{1} F_{1}\left[\frac{1-\eta}{2} ; \frac{1}{2} ; \frac{q_{x}^{2} L^{2}}{4 \pi^{2}}\right]|T(\alpha)|^{2}\left|T(\beta)^{2}\right|
$$

where $\alpha, \beta$ are the grazing angles of incidence and scattering respectively, $I_{o}$ is the incident beam intensity, $k_{o}$ the incident wave vector, $q_{c}$ the wave vector corresponding to the critical angle of incidence, $\Gamma(x)$ is the gamma function, ${ }_{1} F_{1}(x ; y ; z)$ is the Kummer function, and $\mathrm{T}(\alpha)$ is the Fresnel transmission coefficient for incident angle $\alpha$,

$$
\eta=\frac{1}{2} \mathrm{~Bq}_{\mathrm{z}}^{2}
$$

and

$$
\sigma_{\mathrm{eff}}^{2}=\sigma^{2}+\frac{1}{2}(0.5772) \mathrm{B}-\frac{1}{2} \mathrm{~B} \ln (2 \pi / \mathrm{KL})
$$

$\mathrm{L}$ is the coherence length of the beam along the surface or the inverse of the resolution width in $\mathrm{q}_{\mathrm{x}}$ space. For $\mathrm{q}_{\mathrm{x}}<$ the resolution function width, this saturates and merges into the nominal "specular" reflectivity. For larger $q_{x}$, this has the asymptotic form

$$
\mathrm{I}\left(\mathrm{q}_{\mathrm{x}}, \mathrm{q}_{\mathrm{z}}\right) \sim \mathrm{q}_{\mathrm{x}}^{-\left(1-\eta\left(\mathrm{q}_{z}\right)\right)}
$$

This is analogous to the algebraic decay $\mathrm{q}^{(-2-\eta)}$ of $S(q)$ in a $2 D$ crystal for which the displacement correlations possess logarithmic correlation functions ( $q$ being the lateral

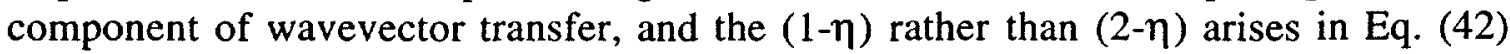


from integrating over $\mathrm{q}_{\mathrm{y}}$ ). In this sense the diffuse scattering around the "specular ridge" in the case of surface scattering is the analogue of the diffuse scattering around the Bragg rods in a 2D crystal. However, in the present case, the exponent $\eta$ is a continuous function of $\mathrm{q}_{2}$, being given by Eq. (40), which can be calculated knowing the surface tension. Experiments carried out with X-ray synchrotron radiation on the surface of liquid ethanol show excellent agreement with the above predictions [43]. (See Fig. 8).

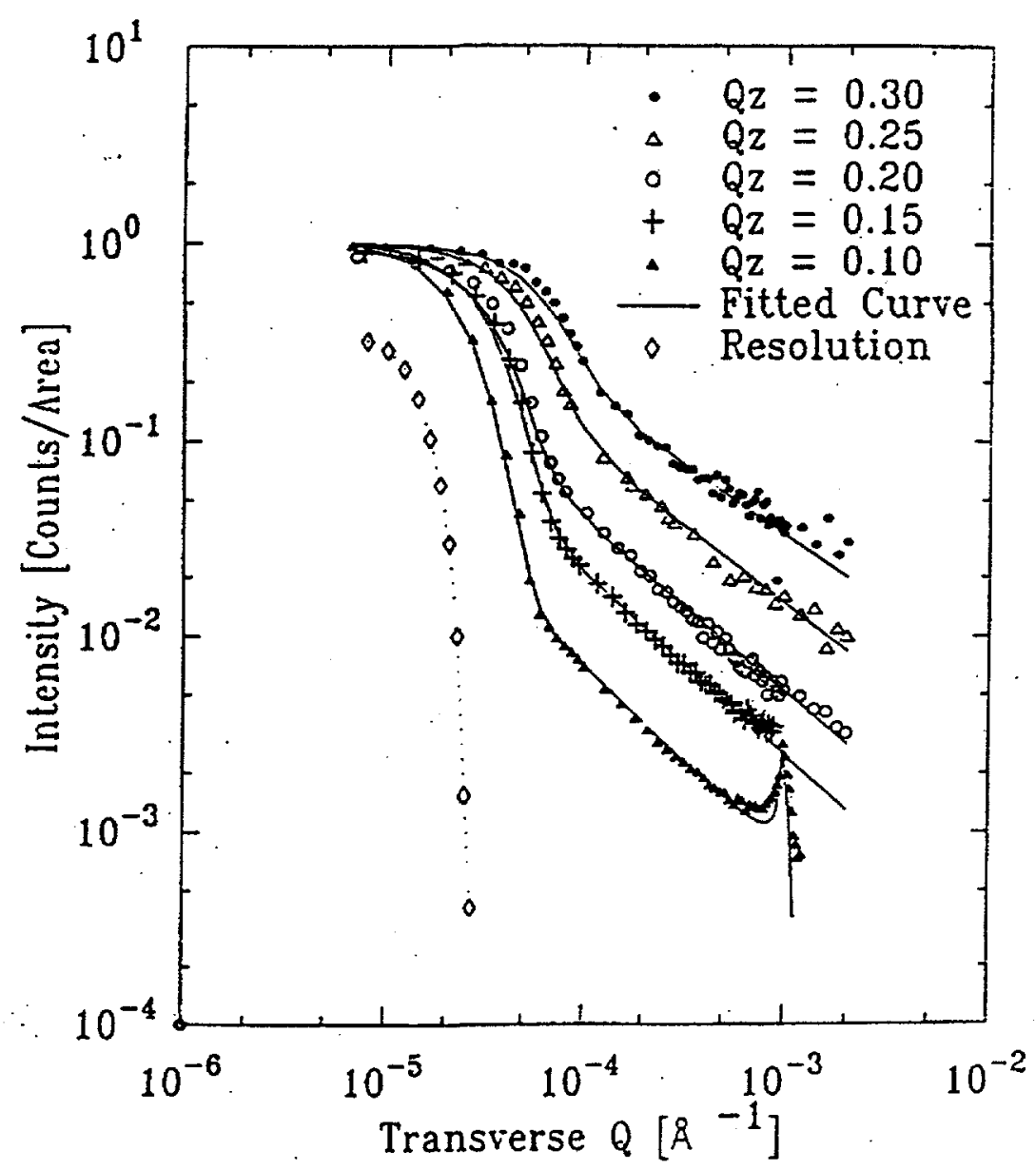

Figure 8. $\log -\log$ plot of the transverse diffuse scattering scans from the surface of liquid ethanol all normalized to unity at $q_{x}=0$ for different values of $q_{z}$. Backgrounds have been subtracted and corrections for variation of illuminated area made. The solid curves represent the calculated scattering. The dashed curve represents the main beam profile converted to an effective transverse resolution at $\mathrm{q}_{z}=$ $0.1 \AA^{-1}$ (From Ref. [43]). 
By Eqs. (38 and 41 ) the effective roughness $\sigma_{\text {eff }}$ measured in a reflectivity experiment is given by

$$
\sigma_{\text {eff }}^{2}=\frac{1}{2} B \ln \left(q_{u} / \Delta q\right)+\sigma_{o}^{2}
$$

where $\Delta q$ is the instrumental resolution width $(2 \pi / L)$ [44]. The so-called "specular" reflectivity from a liquid will be governed by the Debye-Waller-like factor exp $\left(-\mathrm{q}_{z}{ }^{2}\right.$ $\left.\sigma_{\text {eff }}^{2}\right)$ rather than $\exp \left(-\mathrm{q}_{\mathrm{z}}{ }^{2} \sigma^{2}\right)$. The fact that the effective roughness measured for a liquid surface by scattering is less than the true roughness has been known for some time [44] and is due to the unavoidable inclusion of some capillary-wave diffuse scattering inside the resolution broadening of the specular peak. A measurement of $\sigma_{\text {eff }}^{2}$ from specular X-ray reflectivity measurements on liquid alkanes of different chain lengths at different temperatures by Ocko et al [45] (see Fig. 9) yields consistent values for $\mathrm{q}_{\mathrm{u}}$, which appears to scale inversely with the chain length. This leads to the reasonable speculation that the short wavelength cut-off for capillary waves is at a length scale

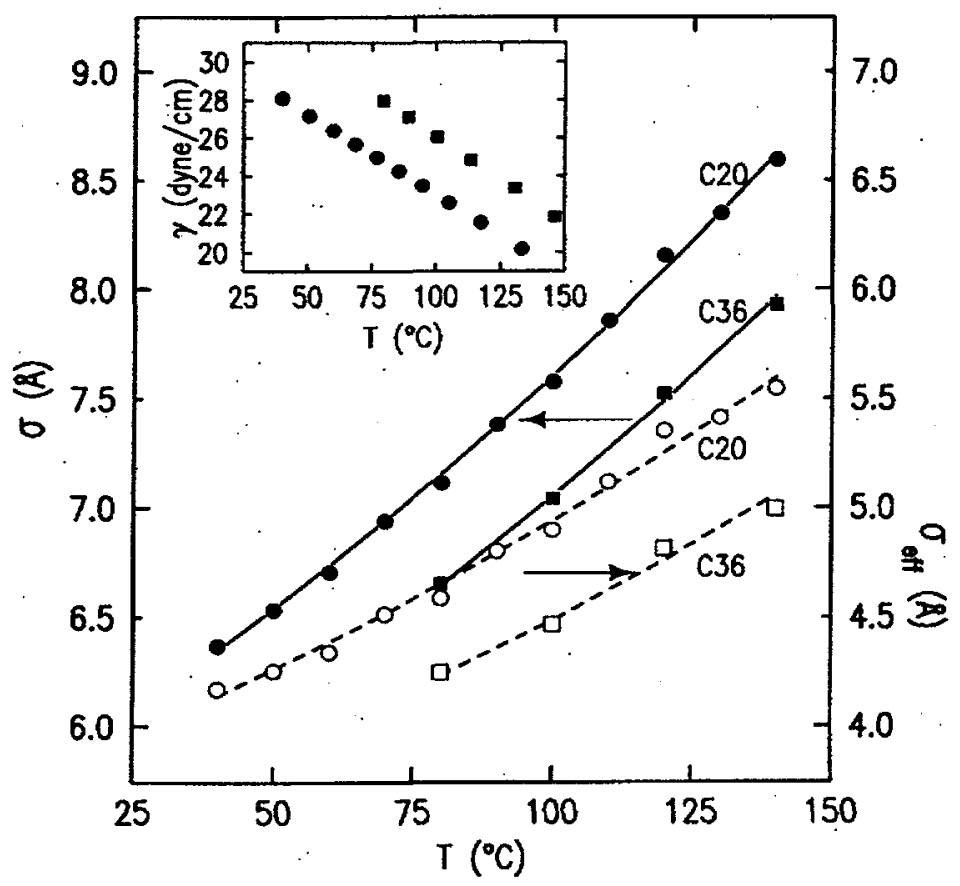

Figure 9. The "true" roughness $\sigma$ and the "effective roughness" $\sigma_{\text {eff }}$ for $\mathrm{C} 20$ and $\mathrm{C} 36$ alkane chains as a function of temperature. The lines are corresponding values calculated with $\sigma_{v}=1.1 \AA, q_{u}=0.44 \AA^{-1}$ for $\mathrm{C} 20$ and $\mathrm{q}_{\mathrm{u}}=0.27 \AA^{-1}$ for C36. This inset shows the measured surface tension $\gamma$ of C20 (circles) and C36 (squares). (From Ref. [45]) 
corresponding to the intermolecular spacing, as in the Debye cut-off for phonons in crystals, although a rigorous proof is lacking. Mode-coupling theory yields such a cutoff naturally by introducing a $\mathrm{q}^{4}$ term in the denominator of Eq. (33), which yields an effective cut-off which is of the same order of magnitude as measured from experiments. We note that such a $\mathrm{q}^{4}$ term in the denominator of Eq. (33) also occurs naturally from a curvature-resisting term in the surface free energy as for a surfactant-covered surface and has been used to fit X-ray scattering from such surfaces [46].

For thin liquid films, the Van-der-Waals interaction with the substrate can enormously increase the value of $\kappa$ defined in $\mathrm{Eq}$. (33), which is now given by

$$
\kappa^{2}=\mathrm{A} / 4 \pi \gamma \mathrm{d}^{4}
$$

where $A$ is the Hamaker constant for the Van der Waals interaction and $\mathrm{d}$ is the film thickness. In such cases, $\kappa$ may actually become larger then the resolution-width $\Delta q$ and a distinct shoulder is seen in the capillary wave diffuse scattering at a value of $q_{x} \sim \kappa$. This is seen in Fig. 10, which shows diffuse scattering (transverse diffuse scans as a function of $q_{x}$ ) from a thin polystyrene film on a silicon substrate [47].

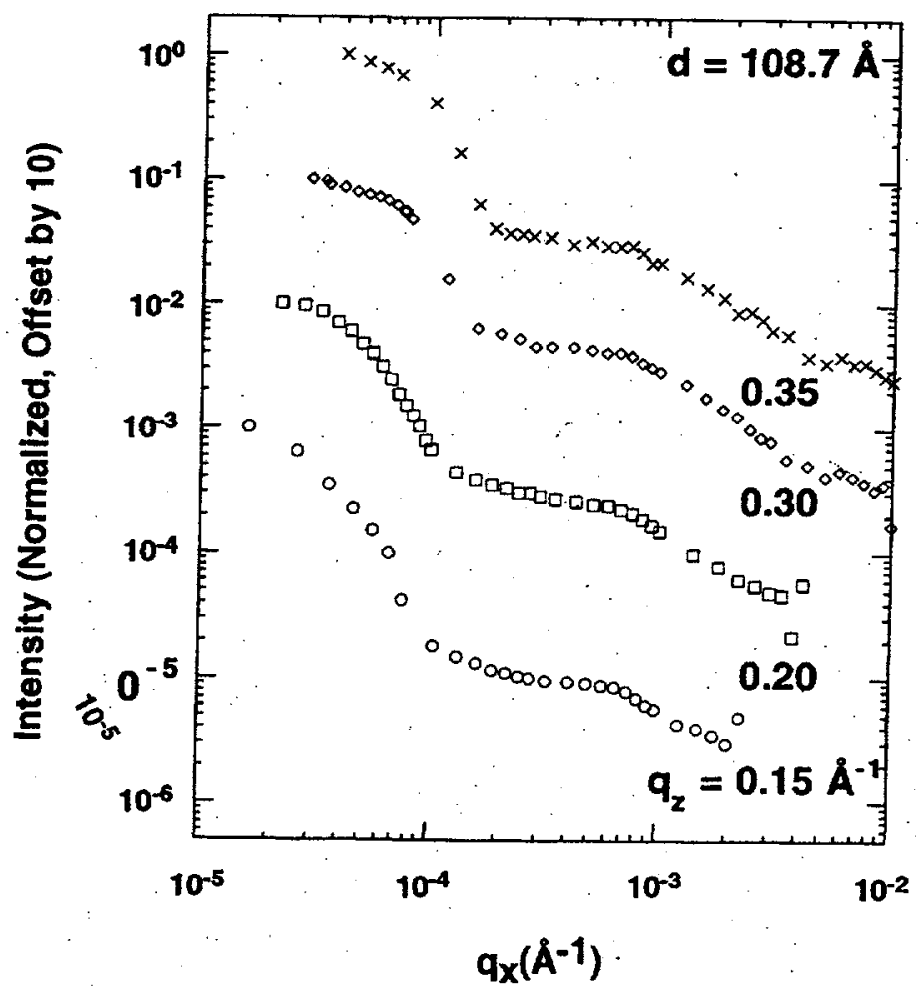

Figure 10. Transverse diffuse scans measured for a $108.7 \AA$ film of polystyrene on a silicon substrate for different values of $\mathrm{q}_{\mathbf{z}}$ showing the finite thicksness cut-off outside of the instrument resolution which is the width of the specular peak. (From Ref. [21]) 
Finally, we discuss briefly the effect of scattering with coherent X-ray beams. For conventional X-ray and neutron sources, the coherence length of the radiation falling on the sample is rather small (typically less than a micron in the directions transverse to the beam, and a few hundred angstroms in the longitudinal direction). This means that the sample scatters from a large number of incoherently scattering volumes, which results in the conventional statistical averages which appear in the correlation functions determining the scattering. However, if the beam is highly monochromatic and highly collimated and the sample is a long distance from the source (which is small in size), as is the situation at the present-day third generation synchrotron sources, then the coherence volumes can be of the order of tens of microns in size and the whole sample can scatter coherently. In general, due to interference effects, disorder or fluctuations in the atomic positions then results in the phenomenon of speckle (familiar to people working with optical lasers) in the scattered beam. As the atoms fluctuate in time, these speckles move in and out of the detector yielding a time-dependent scattered intensity. The auto-correlation function of this intensity can be used to infer the (slow) dynamics of the system. This is completely analogous to the technique of dynamical light scattering used for studying dynamical fluctuations in liquids, but the length scales studied can be much smaller (since $\overrightarrow{\mathrm{q}}$ is larger) and optically opaque systems can be studied. For a system of independently diffusing scatters executing Brownian Motion, e.g., colloidal particles in solution, one obtains

$$
\langle\mathrm{I}(\mathrm{t}) \mathrm{I}(\mathrm{t}+\tau)\rangle /\left\langle\mathrm{I}^{2}\right\rangle=1+\mathrm{Ag}_{2}(\tau)
$$

where $\mathrm{A}$ is a prefactor that can be as large as unity for a perfectly coherent beam, but is often smaller, and $g_{2}(\tau)$ is given by $\exp \left(-2 D q^{2} \tau\right)$, where $D$ is the diffusion constant, which may be q-dependent for larger values of $q$. Such kinds of studies have been performed on colloidal, micellar and polymeric systems [48-51].

I wish to acknowledge my many collaborators in many of the above studies. They include L. Auvray, M. Deutsch, K.G. Huang, J. Lal, M.Y. Lin, B. Ocko, W. Press, M.H. Rafailovich, M.K. Sanyal, O. Seeck, E.B. Sirota, J. Sokolov, P. Thiyagarajan, M. Tolan, J. Wang, X.L. Wu, and X.Z. Wu.

\section{References}

1. Guinier, A. and Fournet, D.C. (1955) Small-angle Scattering of X-rays, Wiley, New York.

2. Glatter, O. and Kratky, O. (1982) Small-angle X-ray Scattering, Academic Press, New York. 
3. Russell, T.P. (1991) Small-angle Scattering at Synchrotron Radiation Sources, in Handbook of Synchrotron Radiation, Vol. 3, (G. Brown and D.E. Moncton, Eds.) North Holland, Amsterdam.

4. Feigin, L.A. and Svergun, D.I. (1987) Structure Analysis by Small Angle X-ray and Neutron Scattering, Plenum Press, New York.

5. Bacon, G.E. (1975) Neutron Diffraction (3rd edition) Clarendon Press, Oxford.

6. Squires, G.L. (1978) Introduction to the Theory of Thermal Neutron Scattering, Cambridge University Press.

7. Brochard, F. and de Gennes, P.G.,(1983) J.Phys.Lett. 44, L-785.

8. Andelman, D. and Joanny, J.F. (1985) in Scaling Phenomena in Disordered Systems, (ed. R. Rynn and A. Skjeltorp) Plenum, New York, p.163.

9. Goldberg, W.I. (1985) in Scaling Phenomena in Disordered Systems, (ed. R. Pynn and A. Skjeltorp) Plenum, New York, p.151.

10. Goh, M.C., Goldburg, W.J., and Knobler, C.M. (1987) Phys.Rev.Lett. 58, 1008.

11. Lin, M.Y., Sinha, S.K., Drake, J.M., Wu, X.L., Thiyagarajan, P., and Stanley, H.B. (1994) Phys.Rev.Lett. 72, 2207.

12. Dierker, S.B. and Wiltzius, P. (1987) Phys.Rev.Lett. 58, 1865 (1989); ibid 62, 804 (1991); ibid 66, 1185.

13. Frisken, B.J. and Cannell, D.S. (1992) Phys.Rev.Lett. 69, 632.

14. Frisken, B.J., Cannell, D.S., Lin, M.Y., and Sinha, S.K. (1995) Phys.Rev.E 51, 5866.

15. Liu, A.J. and Grest, G. (1991) Phys.Rev.A 44, R7894; Monette, L., Liu, A.J., and Grest, G. (1992) Phys.Rev.A 46, 7614; Liu, A.J. et al. (1990) Phys.Rev.Lett. 65, 1897.

16. Maher, J.V., Goldberg, W.I., Pohl, D.W., and Lenz, M. (1984) Phys.Rev.Lett. 53, 60 .

17. Debye, P., Anderson, H.R., and Brumberger, H. (1957) J.Appl. Phys. 28, 679.

18. Wong, P.-z., Cable, J.W., and Dimon, P. (1984) J. Appl. Phys. $55,2377$. 
19. de Gennes, P.G. (1985) Scaling Concepts in Polymer Physics, Cornell University Press, Ithaca, NY, 95.

20. Lal, J., Sinha, S.K., and Auvray, L. (1997) J. de Physique II,7, 1597.

21. Stauffer, D. (1985) Introduction to Percolation Theory, Taylor \& Francis, London and Philadelphia.

22. Daoud, M. and de Gennes, P.G. (1977) J. de Physique 38, 85.

23. Brochard, F. and de Gennes, P.G. (1979) J. de Physique 40, L-399.

24. Als-Nielsen, J., Christensen, F. and Pershan, P.S. (1984) Phys. Rev. Lett. 48, 1107; Wu, E.S. and Webb, W.W. (1973) Phys. Rev. A 8, 2077; Als-Nielsen, J. (1985) Z. Phys. B 61, 411.

25. Born, M. and Wolf, E. (1975) Principles of Optics, 5th Ed. (Pergamon, Oxford), p. 51.

26. Parratt, L.G. (1954) Phys. Rev. 95, 359.

27. Sheng, P. (1976) Phys. Rev. Lett. 37, 1059; Miyano, K. (1979) Phys Rev. Lett. 43, 51; Allender, D. W., Henderson, G.L. and Johnson, D.L. (1981) Phys. Rev. A. 24, 1086.

28. Pershan, P.S., Braslau, A., Weiss, A.H., and Als-Nielsen, J. (1987) Phys. Rev. A 35, 4800 .

29. Ocko, B.M., Braslau, A., Pershan, P.S., Als-Nielsen, J., and Deutsch, M. (1986) Phys. Rev. Lett. 57, 94.

30. Ocko, B.M. (1990) Phys. Rev. Lett. 64, 2160.

31. van der Veen, J.F. and Frenken, J.W.M. (1986) Surf. Sci. 178, 382; Ohnesorge, R., Lowen, H., and Wagner, H. (1991) Phys. Rev. A 43, 2870; Oxtoby, D.W. (1990) Nature 347, 725.

32. Holyst, R. (1992) Phys. Rev. B 46, 15542.

33. Wu, X.Z., Sirota, E.B., Sinha, S.K., Ocko, B.M., and Deutsch, M. (1993) Phys. Rev. Lett. 70, 958; Ocko, B.M., Wu, X.Z., Sirota, E.B., Sinha, S.K., Gong, O., and Deutsch, M. (1997) Phys. Rev. E 55, 3164. 
34. Wu, X.Z., Ocko, B.M., Sirota, E.B., Sinha, S.K., Deutsch, M., Cao, B.H., and Kim, M.W. (1993) Science 261, 1018.

35. Deutsch, M., Wu, X.Z., Sirota, E.B., Sinha, S.K., Ocko, B.M., and Magnussen, O.M. (1995) Europhys. Lett. 30, 283.

36. Wu, X.Z., Ocko, B.M., Tang, N., Sirota, E.B., Sinha, S.K., and Deutsch, M. (1995) Phys. Rev. Lett. 75, 1332.

37. Bhushan, B., Israelachvili, J.N., and Landman, U. (1995) Nature 374, 607; Granick, S., (1991) Science 253, 1373.

38. Yu, C, J., Richter, A.G., Datta, A., Durbin, M.K. and Dutta, P. (1999) Phys. Rev. Lett. 82, 2326.

39. Cieplak, M., Smith, E.D., Robbins, M.O., (1994) Science 265, 1209; Diestler, D.J. Schoen, M., and Cushman, J.H. (1993) Science 262, 545.

40. Demirel, A.L., and Granick, S. (1996) Phys. Rev. Lett. 77, 4330.

41. Napiorkowski, M. and Dietrich, S. (1993) Phys. Rev. E 47, 1836.

42. McClain, B.R., Lee, D.D., Carvalho, B.L., Mochrie, S.G.J., Chen S.H., and Litster, J.D. (1994) Phys. Rev. Lett. 72, 246.

43. Sanyal, M.K., Sinha, S.K., Huang, K.G., and Ocko, B.M. (1991) Phys. Rev. Lett. 66, 628 .

44. Braslau, A., Pershan, P.S., Swizlow, G., Ocko, B.M., and Als-Nielsen, J. (1988) Phys. Rev. A 38, 2457; Meunier, J. and Langevin, D. (1982) J. Phys. (Paris) Lett. 43, L185.

45. Ocko, B.M., Wu, X.Z., Sirota, E.B., Sinha, S.K., and Deutsch, M. (1994) Phys. Rev. Lett. 72, 242.

46. Fradin, C., Daillant, J., Braslau, A., Luzet, D., Gourier, C., Alba, M., Grubel, G., Vignaud, G., Legrand, J.F., Lal, J., Petit, J.M., and Rieutard, F. (1997) Proceedings of 5th International Conference on Surface X-ray and Neutron Scattering (SXNS-5), J. Penfold and D. Norman, Eds., Physica. B (to be published).

47. Wang, J., Tolan, M., Sood, A.K., Wu, X-Z., Li, Z., Bahr, O., Rafailovich, M.H., Sokolov, J. and Sinha, S.K. (to be published). 
48. Dierker, S,B., Pindak, R., Fleming, R.M., Robinson, I.K., and Berman , L. (1994) Phys. Rev. Lett. 73, 82.

49. Thurn-Albrecht, T., Steffen, W., Patkowski, A., Meier, G., Fischer, E.W., Grubel, G., and Abernathy, D.L. (1996) Phys. Rev. Lett. 77, 5437.

50. Mochrie, S.G.J., Mayes, A.M., Sandy, A.R., Sutton, M., Brauer, S., Stephenson, G.B., Abernathy, D.L. and Grubel, G. (1997), Phys. Rev. Lett. 78, 1275.

51. Grubel, G., et al. (1994) ESRF Newsletter, Vol. 20, P. 1. 